\title{
L'Habiter durable : du détournement philosophique à travers les images et les récits médiatiques
}

Véronique André, Laurent Couderchet et André-Frédéric Hoyaux

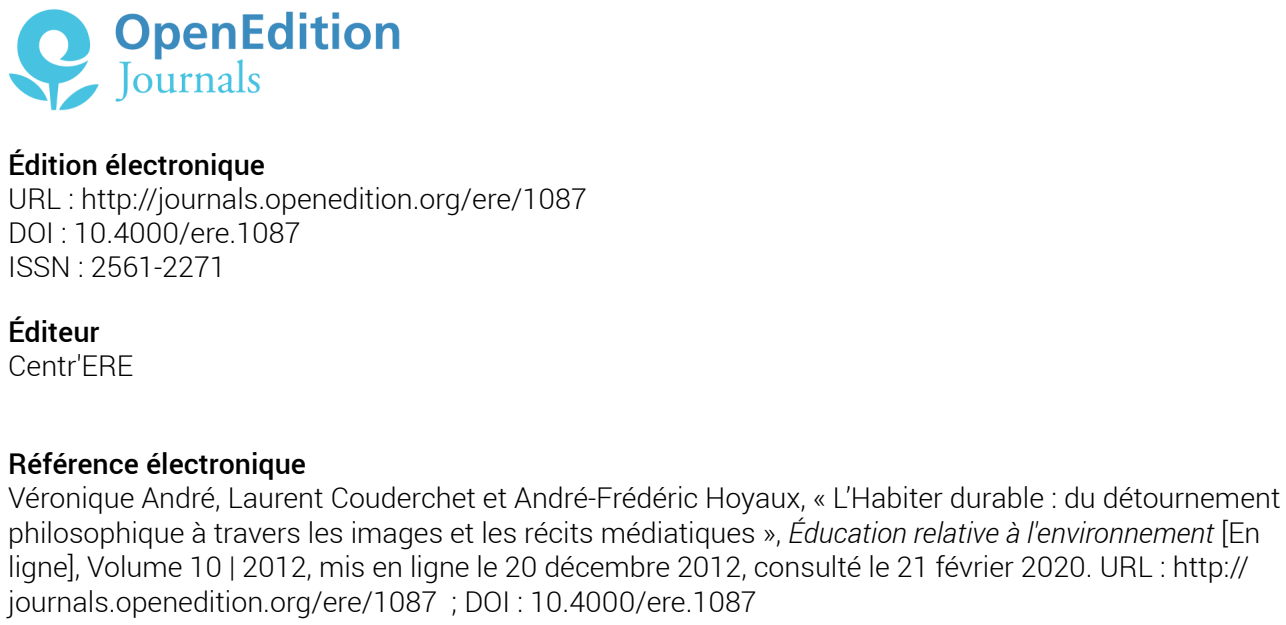

Ce document a été généré automatiquement le 21 février 2020. 


\title{
L'Habiter durable : du
} détournement philosophique à travers les images et les récits médiatiques

\author{
Véronique André, Laurent Couderchet et André-Frédéric Hoyaux
}

Si le concept d'habiter se nourrit à la fois de la relation de l'homme à l'espace et de l'homme à lui-même à travers cette relation à l'espace, ces relations invitent le chercheur à travailler simultanément sur deux champs d'investigations phénoménologiques éprouvées par la philosophie heideggérienne et qui sont utiles à la géographie. Celui de « déloignement » (qui recouvre en fait l'idée de mise à proximité), renvoyant aux concepts de coprésence (proximité réelle: deux personnes ou une personne et un objet sont à côté l'un de l'autre) et de cospatialité (proximité technicisé ou imaginé : deux personnes ou une personne et un objet sont mis en proximité à travers un objet technique ou par la pensée) travaillés par Jacques Lévy et Michel Lussault (2003), et celui d'authenticité, renvoyant au concept d'identité à la fois dans son versant socio-psychologique développé entre autres en géographie par Guy Di Méo (1998), mais aussi dans son versant culturel lié à la nostalgie d'une nature originelle.

2 Avoir travaillé l'habiter des phénoménologues (Buttimer, 1980 ; Hoyaux, 2002 ; Paquot, Lussault et Younès, 2007) permet d'appréhender la récupération de ces deux concepts de déloignement et d'authenticité, et de l'ensemble du dispositif philosophique de la phénoménologie heideggérienne (la question du projet, de l'incarnation, du corps, du monde, de la technique, du quotidien) par les sciences humaines et sociales traitant de l'organisation de l'espace (géographes, urbanistes), et plus globalement par la sphère publique, s'intéressant aujourd'hui au développement durable et à l'écologie. De nouvelles façons d'envisager l'avenir de nos territoires recouvrent en effet un ensemble de lieux communs autour de la nécessité de faire ou de mettre proche, de faire ou de construire authentique, soit à travers l'imaginaire de la ville compacte censé permettre tout à la fois des économies d'énergie et de nouvelles formes de socialité entre les 
habitants des villes, soit à travers l'imaginaire d'un retour à la nature, recouvrant et permettant autant de jeux métaphoriques entre la nature de la Nature (Morin) et la nature de l'Être (Hegel, Heidegger), constituant majeur de l'ontologie heideggérienne.

Pour en prendre conscience, cette contribution utilisera les éléments médiatiques comme porteurs de cette nouvelle rhétorique sociale commune. Parce que la publicité a du sens commun, mais surtout utilise le «bien pensé » supposé de ce sens commun, elle apparait encore plus pertinente que la traduction des écrits scientifiques ou politiques en la matière. Car si la publicité s'attaque à un champ référentiel, c'est que la société consommatrice est prête à en appliquer les diktats, et cela avec subtilité et humilité : faire croire qu'elle est force de médiatisation, non de proposition ni de décision, les deux devant revenir à l'habitant lui-même. Cette démonstration se fera à travers l'analyse des mises en scènes et en récits de publicités vantant les mérites du développement durable et de la " protection » de l'environnement.

Autour de la réutilisation des notions et concepts de la phénoménologie, l'idée est surtout de montrer que la sphère économique est toujours dans l'adaptation idéologique et qu'elle a une grande capacité à nourrir l'incorporation sémantique des habitants, c'est-à-dire de leur proposer des discours prêt-à-porter, mais de leur faire croire en même temps que ce prêt-à-porter est paradoxalement totalement singulier et au-delà qu'ils en sont les porteurs (au double sens du terme).

5 Cette nouvelle rhétorique insidieuse tourne autour des référents du green washing (vert, forêt, campagne) qui sont censés faire nature donc renvoyer à la nostalgie des origines, celle qui justifie à elle seule la meilleure intégration de l'homme sur la terre, de l'être-au-monde : Soyez les premiers à trouver votre ORIGIN ${ }^{1}$ (du nom d'une opération de logement réalisée par Nexity à Bordeaux).

Elle se complait aussi des nouvelles formes de mobilités dites écologiques en quête de coprésence immédiate ou projetée :

En devenant la première entreprise au monde à s'équiper de 500 véhicules électriques de nouvelle génération, La Poste agit au quotidien pour respecter l'environnement. Et quand La Poste s'engage contre le changement climatique, c'est pour que son action se ressente près de chez vous comme à l'autre bout du monde. (Publicité La Poste)

Elle s'arrange également de la seule co-spatialité promise par le téléphone portable, et Internet, traduisant cet esprit ubiquiste cher au phénoménologue (Hoyaux, 2005) :

Vous êtes partout chez vous. (Publicité sur le i-modeTM de Bouygues Télécom)

Si vous êtes parti de chez vous en oubliant d'éteindre la lumière et de protéger la

couche d'ozone. Vous pourrez le faire de votre travail. (Publicité France Telecom)

Toutes ces publicités voudraient faire admettre que la cause est entendue, l'innovation est en marche, et en la matière, l'écologie est le nouvel élément structurant de l'habitation durable du Monde. Car l'écologie serait le ferment idéologique au déclin du tout déplacement (question de l'éloignement, et donc de la coprésence et de la cospatialité), mais aussi d'un retour symbolique à une meilleure symbiose avec la nature, notre nature et une meilleure adéquation de nos techniques face aux enjeux d'un nouveau « contrat naturel » (Serres, 1999). «Donner à la nature les moyens de se faire entendre » (publicité Bouygues).

9 Et il n'y a pas mieux pour un publicitaire aujourd'hui que de se plonger dans la rhétorique phénoménologique sur l'habiter, celle qui en appauvrissant son message initial lui fait dire et nous fait croire qu'il faut «habiter en poète" pour reprendre 
l'expression chère à Hoelderlin, travaillée par Heidegger (1958). Habiter en poète relevant de cette idée multidimensionnelle que chacun d'entre-nous constitue son monde et que l'assignation qui nous est faite par et dans la société relève de notre responsabilité créatrice, innovante, éthique au regard de la durabilité de ladite société. Car chaque habitant invente son monde et c'est à lui de le configurer. «On peut espérer un monde plus écologique, et aussi décider de l'inventer»(publicité EDF).

\section{Reformater l'habiter : Une utopie des politiques et de leurs affidés}

10 On peut s'interroger sur ce qui a réellement changé dans cette habitation. Car, quelles que soient les générations d'architectes et urbanistes qui se sont succédé, elles ont toujours été éprises d'un sentiment de supériorité idéologique. Chaque révolution, chaque nouvelle modernité dans la construction de l'espace se sont fondées sur l'idée que la refonte des qualités de vie architecturales et urbaines conditionnerait de fait le bien-être de celles et ceux qui y vivent! Pour autant, cette évolution s'est surtout structurée sur des récits plus ou moins magiques (Bofill, 1989 ; Le Corbusier, 1957) qui ne cachent d'ailleurs plus la réalité des faits en la matière aujourd'hui. Vanté par ces faiseurs de miracles, le bien-être supposé des grands ensembles modernes ou de certaines banlieues résidentielles s'est en effet brisé sur la réalité vécue par les habitants (Caron, 2010; Degoutin, 2006). Pour autant, la plupart des magazines municipaux des principales agglomérations françaises exposent, toujours à grand renfort de publicités plus ou moins maquillées en informations, cette rhétorique chamanique de l'innovation urbaine attachée au bien-être individuel des populations. Cette démarche autour des Bassins à flot constitue aujourd'hui l'exemple d'un nouvel urbanisme, plus orienté vers le sur-mesure et le respect du génie du lieu (Bordeaux).

11 Ainsi, la question se pose surtout sur le sens réel de cette nouvelle mise en récit du monde à travers l'habitation écologique. Et en cette occasion, n'est-ce pas juste réduire l'habiter à une nouvelle forme de résidentialité pour une classe moyenne supérieure urbaine ayant les moyens de se placer symboliquement, à travers cette innovation, dans les strates sociales de la société contemporaine? Car, outre les moyens économiques que certains n'ont pas pour mettre en œuvre ce nouveau récit, il est intéressant de se demander si fondamentalement cet écologisme fait sens pour cette population. Et au-delà, qui possède la mainmise sur ce sens : est-ce les publicitaires euxmêmes, qui par pure stratégie commerciale, amènent leur commanditaire sur ce segment écologiste? Est-ce les institutions qui, poussées par la hiérarchisation des décisions, se sentent vouées à faire des efforts en la matière pour répondre aux décisions, normes, lois nationales ou internationales? Est-ce l'imaginaire ambiant collectif de la nation française, européenne, mondiale qui, pris de peur pour l'avenir de ses enfants, prend conscience de l'impasse dans lequel notre façon d'habiter le monde se trouve?

Il semble intéressant sur ces questionnements de comprendre le sens que les habitants donnent à leur ancrage territorial et en quoi habiter est aussi un moyen de justifier ce que l'on est dans le monde, ce que l'on fait avec les éléments qui le structurent et les idéaux qui le fondent. Mais le sens donné par les habitants est aussi une mise en lumière des conformations liées à l'éducation, des fameuses incorporations chères à Bourdieu (1994). L'habitant vit en effet aussi des artefacts de mondes qu'il se configure 
face à lui visuellement (paysage « réel » et « virtuel») à travers les déplacements qu'il effectue dans le monde, mais aussi à partir des publicités qu'il ingurgite à longueur de journée. Il est alors persuadé que ce qu'il se projette intellectuellement et qui le construit mentalement, est de l'ordre du donné, du naturel, du non discutable, de «l'allant de soi » (Schütz, [1942-1966] 2007) alors que ce n'est qu'un artifice, qu'une fiction que l'extérieur lui envoie parfois sous couvert d'un marketing publicitaire plus ou moins manipulé par des lobbies (Staulber et Rampton, 2004). En effet, comme tout discours, le discours écologique est légitime pour celui qui le porte et il se nourrit de valeurs de qualifications et de justifications (il faut utiliser des ampoules basse consommation parce que..., il faut faire du covoiturage parce que..., il faut arrêter son moteur au feu rouge parce que... la nature en a besoin) (Lussault, 2003, p. 39-42). Audelà de sa propre identité qu'il se construit à travers son habitation, on lui impose un peu partout, par des incitations économiques et des messages politiques, des façons d'être, de faire et de penser réifiés, chosifiés qui n'ont plus rien à voir avec la liberté d'habiter, mais qui relèvent peu ou prou d'une propagande (Bernays, [1928] 2007).

À partir du moment où les dirigeants politiques et économiques des sociétés contemporaines ont compris, notamment à la suite des travaux phénoménologiques, que l'être humain à haut capital économique et culturel pouvait constituer, au sens plein du terme, son monde, l'enjeu est devenu pour ces dirigeants de reformater ces imaginaires constitutifs. Cet enjeu prendrait donc acte que ce ne sont plus les contraintes naturelles, économiques, historiques, sociales qui déterminent les actions de l'être humain, ses possibles, mais qu'il peut à tout le moins les détourner pour partie et inscrire les aspects volitifs de ses intentionnalités, de ses projets. Ainsi, pour guider les intentionnalités humaines vers des intentions nouvelles qui se traduiraient in fine dans des actes quotidiens, il faut construire de nouveaux artefacts qui amèneront cet être humain à changer ses pratiques et à trouver son bonheur dans la justification de ces nouvelles actions.

L'objectif est donc de faire croire à l'être humain qu'il est libre de ses actes et de la pensée de ses actes, mais aussi que chaque acte qu'il pose lui permet de se placer, c'està-dire de s'auto-assigner une position spatiale et de s'auto-désigner une situation sociale au sein du monde. Cette place est en quelque sorte co-extensive à la corporalité (en tant qu'entité physique) de l'être humain, c'est-à-dire qu'elle dépasse son enveloppe physique pour s'imposer d'autres dimensions dans l'espace à travers ce que les phénoménologues appellent la corporéité, cette capacité de la chair à dépasser l'imaginaire euclidien de la distance étendue et de la distance temps, pour entrevoir le jeu plaçant des métriques. Aller au-delà pour être proche de celles et ceux avec qui on a envie d'être; agir autrement pour s'identifier à d'autres codes, normes, rôles que ceux que l'être humain devrait tenir a priori. Cela nous amène à éclairer, dans un premier temps, le dispositif conceptuel de l'habiter sur lequel se fonde aujourd'hui ce marketing et, dans un second temps, de voir les implications pratiques utilisées par la sphère médiatique.

\section{Habiter : au départ était le projet de prendre chair}

Pour les phénoménologues, dès sa naissance, l'être humain est "toujours déjà » en relation avec un monde, il est être-au-monde, les tirets exprimant cette relation qui ne s'achève qu'à sa mort. Par cette particularité, Heidegger insiste sur l'indéfectibilité du 
lien et de l'interrelation qui se réalise entre l'être humain et la terre où il s'incarne, où il prend corps en tant que chair et conscience dans un lieu et un temps donnés. En un sens chrétien, il prend pleinement, corps et âme, sa place dans le monde.

"L'homme est dans la situation d'être-jeté » (Heidegger, [1946] 1953-1983, p. 109). Mais ce n'est pas l'homme qui se jette, c'est «l'Être lui-même qui destine l'homme à l'eksistence de l'être-le-là comme à son essence " (Heidegger, [1946] 1953-1983, p. 97). L'homme est bien une singularité qui exprime par le fait d'être un là dans un lieu et un temps donnés, une compréhension singulière d'un Être universel, Être qui se doit de s'ouvrir à sa singularité en se projetant dans un monde. Ce projet (pro-jet : jeter en avant de, à terre) est la signification du «ek» de ek-sistence. L'être humain a pour nécessité « de se jeter en avant de » l'Être et de «se tenir debout » (sistence - du latin sistere) dans un monde. Un monde spatial, mais aussi social. Un monde de significations qui artefactualise toute réalité, mais qui n'épuise jamais l'émergence du sens et sa totale substance.

De ce fait, l'être-au-monde ne peut être conçu pour l'habitant que comme une configuration constituante et constitutive de ce qu'il est, croit, pense. Il s'en remet à elle pour justifier à toutes fins utiles que ce soit philosophiquement, psychologiquement, socialement, etc. de l'opérativité de sa compréhension du monde et de lui-même. Si pour les philosophes, l'être-au-monde est un concept, une sorte d'objectité, pour le géographe travaillant avec des habitants pensant leur monde, c'est un horizon ontologique qu'ils convoquent pour donner sens à ce qu'ils sont, font, et pensent dans, sur et en ce monde.

Et si le sens de cette configuration projetée face à lui à travers l'environnement où il se trouve peut dire autant que lui-même, si l'espace de vie peut consubstantiellement parler pour celui qui y vit, alors habiter est bien «le bonheur incarné » (publicité sur une opération de logement réalisée par Nexity à Bordeaux). L'intérêt est alors de comprendre comment l'habitant justifie la construction dialectique de mise à proximité versus mise à distance (dite construction territoriale) par l'utilisation de référents moraux ou idéologiques, c'est-à-dire par la constitution ontologique d'un système de valeurs qui détermine l'interprétation des phénomènes qui se déroulent en ce monde et la compréhension ontologique qui est faite de ce monde par et pour lui. Ces deux questions qui recourent aux deux tournants géographiques (épistémologiques et méthodologiques), amène à prolonger notre étude sur deux points abordés par la phénoménologie, celui du déloignement et celui de l'authenticité, l'un référant à la proxémie, à la coprésence ; l'autre à la construction identitaire.

\section{Habiter : Un monde de mises à proximité et de mises à distance}

19 Suivre la phénoménologie de l'espace (Chrétien, 1983 ; Franck, 1986), c'est renverser les prérogatives géographiques habituelles dans l'analyse des relations de l'être-au-monde. Il n'y a plus de contenant, de conditionnant, de catégorie qui détermine a priori les attributs de ce dernier. Au contraire, c'est dans son souci d'être-là et justement d'avoir tels attributs afférents à sa finalité d'être-au-monde que le monde de l'être est tel qu'il est, en son contenu (le sens), en ses potentialités (les projets). 
20 L'analyse demande de déconstruire le monde de l'être-au-monde, en tant qu'entité formalisée et délimitée spatialement, entité préconfigurée dans laquelle l'être-là qui est au monde viendrait exister, se projeter. Cette analyse appelle à déconstruire les distances objectives comme instigatrices a priori des relations privilégiées de cet êtreau-monde avec les éléments de ses entours (espaces de proximité au sens de l'étendue) et de ses contrées lointaines. Il faut plutôt concevoir que ce sont les mises en relation spatiale et sociale de l'être-au-monde qui déterminent son champ territorial (celui de ses appropriations spatiales et de ses sentiments d'appartenance aux différents collectifs) et les divers éléments ou être-au-monde des entours ou des contrées qui sont ainsi mis à sa proximité. De ce fait, toute relation au monde, spatiale et/ou sociale, exprime la construction territoriale de l'être-au-monde et imprime alors a posteriori la configuration du monde de ce dernier. Cette configuration génère comme elle est générée par la mise en mesure du monde par l'être-au-monde. Cette mesure ne réfère plus alors à une "échelle normale " (distance-étendue euclidienne), mais à sa «métrique particulière » (distance phénoménologique ou corporéique de l'espace).

21 Cette construction territoriale s'établit à partir de la territorialisation qu'engage l'êtreau-monde, du fait même qu'il est toujours-déjà en avant de lui-même dans son monde. Cette territorialisation découle de sa préoccupation (envers des espaces ou des objets dans l'espace) et de sa prévoyance (envers des personnes). L'analyse de ces dernières permet de concevoir des dialectiques de mise à proximité vs mise à distance, qui ne sont pas constitutives, mais bien constituées par l'analyse a posteriori qu'un géographe peut faire. En effet, par la mise à proximité des choses et d'autrui, l'être-au-monde s'approprie des espaces, s'identifie à des personnes, et routinise ses actions, mais il codétermine aussi une mise à distance des autres choses (personnes, espaces, événements).

La publicité va coupler ces deux concepts heideggériens de préoccupations et de prévoyances inhérents aux soucis fondamentaux de l'être-au-monde quand il déloigne son monde.

Remplacer une réunion par une visioconférence c'est aussi protéger un iceberg. [...] Le télétravail, les échanges de données dématérialisées, les communications à distance par visioconférence, sont des exemples de solutions qui permettent d'abolir les distances, tout en gardant les liens et les échanges nécessaires entre différents sites, où que l'on soit. (Publicité France Telecom)

En pensant à mon travail et à celles et ceux avec qui je travaille, je me préoccupe également de la nature, c'est-à-dire que je préserve ce monde des possibles. Pour Heidegger, on entre en relation " avec », donc on met à proximité, uniquement ce que l'on connaît, que ce connu soit valorisé positivement ou négativement. Ce connu est donc une totalité qui ne peut se fractionner. La préoccupation et la prévoyance sont donc toujours, en théorie, englobantes et non excluantes. En effet, mettre à distance, ce n'est pas rejeter hors du monde, c'est au contraire conserver la préoccupation de cette présence, mais la mettre en marge dans un espace que l'on ne veut pas voir comme central en terme de pratiques et de représentations. Car, on ne peut être préoccupé par l'absence, puisque penser à quelque chose ou à quelqu'un d'absent, c'est déjà le rendre à la présence à soi, que cette présence soit entendue comme un manque ou non.

24 Caractérisé de cette façon, le déloignement (Heidegger, [1927] 1964, p.133) est la possibilité offerte à l'être-au-monde de construire son monde, en ce qu'il rapproche à lui son monde spatial, social et temporal, et occulte le reste. C'est le déloignement qui 
exprime explicitement pour l'être-au-monde sa relation à celui-ci. Ce ne sont pas les rapports d'évidence qui font que l'être-au-monde est proche d'une personne, d'un lieu, d'un événement. Le monde, et même le monde objectivement proche, est empli de personnes, de lieux, d'événements, parmi lesquels, pourtant, l'être-au-monde ne retient, par son rapprochement, que certaines personnes, certains lieux ou objets, certains souvenirs.

L'analyse du déloignement montre donc la relativité des rapports à l'espace, au temps et aux personnes qu'entretient l'être avec le monde. En ce sens, il est bien autonome et responsable de ces choix de mise à proximité. Cette relation au monde exprime avant tout un monde de significabilités, qu'entrouvrent à chaque instant (selon une territorialisation) les territorialités de l'être-au-monde. Celles-ci engagent donc toutes les prédications qu'effectue l'être à l'encontre de ses rapports au monde, ceux de la distance spatiale, de la profondeur des relations sociales, de la durée.

Un chemin «objectivement» plus long peut être plus court qu'un chemin « objectivement » très court, si ce dernier est « un véritable calvaire » et s'il paraît à celui qui le parcourt infiniment long. Or, c'est dans un tel «apparaître» qu'un monde est proprement et véritablement disponible. Les distances objectives qui séparent des choses subsistantes ne coïncident point avec l'éloignement et la proximité des étant intramondains disponibles [...] Et si même nous usons d'une mesure plus nette, en disant qu' "il y a une demi-heure d'ici à la maison », cette mesure encore doit être tenue pour une simple évaluation. Une « demi-heure » n'est pas égale ici à trente minutes, mais à une durée dénuée de «longueur ", si l'on entend par longueur une extension quantitative. Cette durée se comprend en termes de "préoccupations " quotidiennes. Même là où il existe des mesures précises et "officielles", l'éloignement s'évalue de prime abord selon la prévoyance. (Heidegger, [1927] 1964, p. 135)

Les distances entre les choses, entre l'être et les choses, ainsi que la durée, correspondent bien aux relations qu'entretient l'être dans sa préoccupation auprès de son monde, que ces relations soient considérées comme bonnes ou mauvaises, trop courtes ou trop longues. Et s'il faut faire de l'énergie écologique, s'il faut densifier les points de fixation de la population pour éviter de démultiplier les réseaux et les coûts éco-énergétiques que cela semble entrainer, il n'en reste pas moins, que les grands groupes énergétiques doivent promouvoir l'équi-habilité des lieux. Celle-ci se structure par le sens que l'être donne à sa place dans le monde, le monde rêvé pour certains d'une nature originelle, celle d'une maison perdue dans l'immensité d'un paysage de montagne vierge de toute autre construction et au milieu duquel une femme erre avec son panier de linge vide dans un champ de vision structuré par un étendage évoquant les fils électriques du premier producteur d'électricité européen: «Faisons le choix d'un monde avec moins de $\mathrm{CO}_{2}$ » (publicité EDF).

Les questions de mise en coprésence à travers les mobilités spatiales ou les mobilisations techniques permises par le téléphone portable, internet, etc. (Hoyaux, 2005) sont ici travaillées par les publicitaires.

Vos proches ne sont jamais loin. La distance entre deux villes compte moins que la distance entre deux êtres. Parce que chaque destination a le visage de quelqu'un qui vous est cher. Parce que la liberté n'a pas d'horaires, nos liens sont faits d'autoroute. Pour être avec ceux qu'on aime, partout où on le veut et à n'importe quelle heure. Que ferions-nous sans autoroute? L'autoroute. Un trait d'union entre nous. (Publicité du Consortium des autoroutes françaises) 
Mais puisque dans nos sociétés contemporaines, il faut faire croire que l'habitant est responsable du monde qu'il constitue face à lui, il est nécessaire de lui donner les armes conceptuelles et pragmatiques pour instituer ce monde : c'est-à-dire transmettre cette idée de responsabilité de la décision et de singularité du choix effectué à cette occasion, même si celui-ci n'intervient pas.

Dans le groupe Carrefour, vous être utile chaque jour, c'est être proche de là où vous êtes. Il suffit de nous dire où. On s'occupe de tout. La proximité d'un constructeur régional, les garanties d'un réseau national. [Avec un Ici en gros caractère à l'endroit présumé de la construction au milieu d'un village campagnard.] (Publicité Maisons d'En France)

Ce passage permet d'ouvrir sur la question des métriques et des jugements de valeurs qui s'y réfèrent. Ces derniers ne sont plus à concevoir comme la mise en place d'une vérité en tant que telle pour l'être-au-monde, c'est-à-dire en tant que ce jugement de valeur pourrait se comprendre en soi, sans le relier à ce pourquoi ce jugement de valeur est justement émis. Tout jugement de valeur est par principe à référer au couple mise à proximité versus mise à distance du monde exprimé par l'être-là lui-même dans la situation où il le formule. Percevant avant terme l'acrimonie probable des scientifiques, Heidegger précise justement la teneur de cette indexation à la situation de l'énonciation.

Pour qui a été formé à la considération de la «nature» et des distances «objectivement» mesurées entre les choses, il y aura risque de tenir pour « subjectives » cette explicitation de l'éloignement et cette manière d'évaluer. Mais il s'agirait alors d'une «subjectivité » qui nous découvre, peut-être, ce qu'il y a de plus réel dans la « réalité » du monde et qui n'aurait rien à voir avec l'arbitraire « subjectif » et les « opinions » subjectivistes touchant un étant qui « en soi » serait autre. C'est la faculté de déloignement, exercée par l'être-là dans sa prévoyance quotidienne, qui nous découvre l'être-en-soi du "monde vrai ", c'est-à-dire de l'étant auprès duquel l'être-là, en tant qu'il existe, est toujours-déjà présent. (Heidegger, [1927] 1964, p. 135)

30 Ainsi, analyser le jugement de valeur en tant que tel n'a aucun intérêt pour le chercheur en sciences humaines et sociales. En revanche, il permet de caractériser le déloignement de l'être-au-monde, par son côté hédoniste notamment. Le propre et le mal-propre, les bonnes et mauvaises odeurs, les ambiances sûres et inquiétantes sont des jugements qui ne sont intéressants que dans la mesure où ils permettent à l'êtreau-monde de configurer un territoire signifiant pour soi et les autres qui coexistent avec lui, mais aussi, au-delà, de comprendre ce pour quoi son territoire est signifié comme tel.

Développer le haut débit et pouvoir s'installer là où on ne s'installait plus. [...] Enclavement des territoires et déficit de l'emploi sont souvent dans les campagnes le pendant de l'asphyxie des villes. (Publicité France Telecom)

31 La relation territoriale est donc avant tout une relation sémantique. Celle-ci permet de configurer le monde de l'être-au-monde à partir notamment des relations indexicales explicitées à partir des indexicalités adverbiales «de lieu» ou «de temps", pronominales «de personne» et verbales «de temps» que cet être-au-monde entretient au travers de ses discours sur le monde. Relations adverbiales, pronominales et verbales qui corroborent l'idée d'un dépassement, d'une extension phénoménologique du corps en tant que je - ici - maintenant par sa projection auprès de choses et avec des êtres. 

sa préoccupation, sa pensée. Le lieu devient lien permanent, appréhension du monde dans sa quotidienne apparition, dans sa quotidienne rencontre. Le territoire est l'immanence du re-tenir, la réitération dans un "résolument ouvert », mais « limité » de ce re-tenir. Quant au monde de l'être-au-monde, il est toujours déjà le «àdécouvrir » dans le découvert. C'est «l'au-delà », ce « il », ces « eux », cet « à-venir » du projet à faire que l'on ramène un jour à soi en tant qu'expression ultime du changement de ce que l'on est. Le monde de l'être demeure donc comme l'horizon irrésolu, le sens même de la quête, le sens même du projet, la transcendance de l'action de l'être-là en tant qu'être auprès du monde, du Monde. Il demande alors que l'homme l'habite.

Vous êtes en ville chez vous. [Avec l'image d'un parc retravaillé pour représenter un lit de deux personnes, espace de l'intimité par excellence.] (Publicité Urbania)

Le monde de l'être peut donc être compris comme une entité transactionnelle et transitionnelle. Transactionnelle car c'est à l'intérieur de celui-ci que se passe le commerce quotidien avec les choses de l'existence de l'être-au-monde. Transitionnelle car d'un côté, il est toujours évolutif, en transition ; et de l'autre, il est permanent au sens où l'être-au-monde a tendance à le fétichiser comme un objet particulier duquel il ne peut se détacher. Par cette dérive sécuritaire, le monde de l'être devient alors un artefact et non plus un projet. Dès lors, il faut proposer à l'être humain un prêt-àporter idéologique qui se traduit par des actions à réaliser, que la publicité et l'ensemble des messages médiatiques essaient de visibiliser, de bonifier, de valoriser pour l'être lui-même. Car ce n'est pas tout de promouvoir un produit ou une action, il faut aussi lui donner le sens qui va justifier et placer correctement celui qui potentiellement va la réaliser.

\section{Habiter : une quête d'authenticité ?}

Poser une réflexion sur l'authenticité, c'est pour Heidegger la possibilité de séparer de manière très moderne une bonne façon d'être et une mauvaise façon d'être-au-monde, une bonne et une mauvaise façon d'habiter. Pour Heidegger, l'authenticité relève de l'acceptation totale de la révélation à soi de ce que l'être-là découvre dans son monde ! En cela, que la découverte du monde soit l'expression d'une volonté de délimitations ou de distinctions qui configurerait le monde de l'être à l'intérieur du Monde ne relève pas en soi d'une inauthenticité pour l'être-là qui est au monde. En revanche, la volonté pour ce dernier de ne pas comprendre les nécessités ontologiques de cette distinction et de cette délimitation peut le devenir. Car l'être-au-monde constitue sa singularité en même temps qu'il constitue son monde. Cette singularité se construit en soi, et avec les autres, elle s'initie dans la dynamique identitaire entre le désir de personnalisation (être seul à être comme on est) et le désir d'identification (ressembler aux autres).

En se préoccupant de ce qu'on a entrepris avec, pour ou contre les autres, on s'inspire constamment du souci de se distinguer d'avec ces autres. Soit que l'on s'efforce seulement d'effacer toute différence avec eux ; soit que l'être-là, se sentant inférieur, cherche dans ses rapports avec eux à les égaler ; soit encore que l'être-là, se plaçant au-dessus des autres, cherche à maintenir ceux-ci au-dessous de lui. La coexistence - bien qu'elle se le dissimule - s'inquiète et se soucie de cette distance. Ce qu'on peut exprimer existentialement en disant que l'être-en-commun existe sous le signe du distancement. Plus ce mode d'être passe inaperçu de l'être-là quotidien, 
plus profondément et plus tenacement il agit sur lui. (Heidegger, [1927] 1964, p. 158-159) avec autrui tend à faire disparaitre son ipséité d'être-là, car il se plonge dans l'être du « on » et prolonge ses interprétations du monde à partir de ce mode de pensée.

Le « on » est donc celui qui, dans l'existence quotidienne, décharge l'être-là. Ce n'est pas tout ; en déchargeant ainsi l'être-là de son être, le " on » complaît à la tendance qui pousse celui-ci à la frivolité et à la facilité. Cette complaisance permet au " on » de conserver, voire d'accroître, un empire obstiné. Chacun est l'autre et personne n'est soi-même. Le "on", qui répond à la question de savoir qui est l'être-là quotidien, n'est personne. À ce " personne ", l'être-là, mêlé à la foule, s'est toujoursdéjà abandonné. Ces caractères ontologiques de la soumission quotidienne à l'emprise des « autres » : le distancement, la moyenne, le nivellement, la publicité, la destitution de l'être et la complaisance définissent la nature "permanente" et immédiate de l'être-là. [...] Sous les modes qu'on vient de nommer, l'ipséité de l'être-là et celle d'autrui ne se sont pas encore trouvées ou se sont perdues. On est sur le mode de la dépendance et de l'inauthenticité. (Heidegger, [1927] 1964, p. $160-161)$

aper, ce serait l'être-là lui-même qui appellerait à cette inauthenticité, qui se complairait dans le "on ». Mais cet appel par la préoccupation et la sollicitude reconduirait quand même la volonté d'être de l'être-là dans la mesure où c'est toujours en dernier ressort ce dernier qui est la finalité de son propre projet d'être. Pour s'en sortir, Heidegger crée une double ipséité, l'une authentique, l'autre inauthentique.

Cette différence entre l'ipséité authentique et l'ipséité inauthentique ne peut pas être prise en compte dans l'interprétation de la construction territoriale de l'être-au-monde ni même dans la compréhension de l'être-là lui-même. Car le "on" est inhérent à l'emprise du monde en commun, qu'on l'accepte ou qu'on le refuse (par le désir d'êtreseul par exemple) puisque ce refus de l'autre ne peut être conçu que comme une volonté de l'être-là de se révéler, de s'ouvrir (à lui-même) par cette mise en retrait, puisque celle-ci co-détermine l'existence de ce envers quoi l'être se met en retrait. Donc, il faut comprendre que cette emprise $d u$ «on » est plus ou moins forte selon la préoccupation de l'être-au-monde lui-même.

En usant des transports en commun ou des services d'information (des journaux par exemple), chacun est semblable à tout autre. Cet être-en-commun dissout complètement l'être-là qui est mien dans le mode d'être d'« autrui », en telle sorte que les autres n'en disparaissent que davantage en ce qu'ils ont de distinct et d'expressément particulier. Cette situation d'indifférence et d'indistinction permet au " on" de développer sa dictature caractéristique. Nous nous amusons, nous nous distrayons, comme on s'amuse; nous lisons, nous voyons, nous jugeons de la littérature et de l'art, comme on voit et comme on juge; et même nous nous écartons des "grandes foules" comme on s'en écarte; nous trouvons «scandaleux» ce que l'on trouve scandaleux. Le "on" qui n'est personne de déterminé et qui est tout le monde, bien qu'il ne soit pas la somme de tous, prescrit à la réalité quotidienne son mode d'être. (Heidegger, [1927] 1964, p. 159)

La publicité et la sphère médiatique dans son ensemble (y compris l'éducation et la culture) sont représentatives de cet esprit $\mathrm{du}$ « on » inauthentique. La publicité doit donc trouver les moyens pour singulariser et donc authentifier l'action et la pensée des individus. De toute façon, la réappropriation du sens ne peut se déléguer à aucune autre personne que soi-même. Cette responsabilité inhérente à tout être-là qui est au monde peut parfois être encombrante quand ce dernier croit ne pas pouvoir se fonder sur des bases idéologiques objectivées pour expliciter le choix de ses actions - d'autant

Éducation relative à l'environnement, Volume 10 | 2012 
plus en ces périodes où le surcroît d'informations (parfois divergentes) tend à complexifier l'arbitrage de tout un chacun. Cette aporie heideggérienne relève de son " désir » de mettre en garde les êtres humains sur leur déresponsabilisation dans l'agir quotidien. Pour lui, l'être-là se cache derrière le «on » en tant que ce « on » n'est pas réfléchi à la base par l'être-là comme un « je » qui choisit ce " on » pour éclairer « son » là dans le monde.

\begin{abstract}
Le « on » a ses propres manières d'être. La tendance caractéristique de l'être-avecautrui que nous avons nommée le distancement se fonde sur le fait que l'être-encommun cherche à imposer tout ce qui est conforme à la moyenne. [...] Le « on » se mêle de tout, mais en réussissant toujours à se dérober si l'être-là est acculé à quelque décision. Cependant, comme il suggère en toute occasion le jugement à énoncer et la décision à prendre, il retire à l'être-là toute responsabilité concrète. Le « on » ne court aucun risque à permettre qu'en toute circonstance on ait recours à lui. Il peut aisément porter n'importe quelle responsabilité, puisqu'à travers lui personne jamais ne peut être interpellé. (Heidegger, [1927] 1964, p. 159-160)
\end{abstract}

Ce passage prédispose l'idée que la responsabilité humaine procède uniquement de la responsabilité de tout un chacun. Pour Ricœur (1985, p.121), «le règne de l'inauthenticité ne cesse, en fait, de rouvrir la question du critère d'authenticité. C'est à la conscience morale que l'attestation d'authenticité est alors demandée ». Celle-ci prend en compte la conception que l'être-là peut se concevoir, à travers sa préoccupation, en tant qu'être-avec-autrui dans un «on", mais en tant qu'il prend conscience du fait que cette participation à ce " on ", et les pratiques ou actions qui s'y rapportent, relèvent de son entière responsabilité, et non de celle du « on » dans lequel il n'est qu'un élément caché ou noyé dans la masse. Mais pour éviter autant que faire se peut que cette prise de responsabilité ne nuise à la sécurité ontologique (Giddens, 1987) de l'être-au-monde, il faut lui donner confiance en ses moyens et en son intelligence d'habiter de façon innovante et singulière.

Faire grandir la confiance, c'est donner des ailes à chacun. La poste contribue chaque jour au développement de chacun. En modernisant ses bureaux, en développant ses services innovants, en créant la Banque postale et en adoptant une stratégie d'entreprise responsable... (Publicité La Poste)

Ainsi, l'ordre sémantique transparaît dans la morale inscrite depuis l'époque moderne, celle du bien et du mal, du bon et du mauvais, du beau et du laid. Il faut donc que ces valeurs antithétiques fassent en elle-même, par elle-même sens de ce que l'être est. Ce que nous pourrions appeler la tautologie axiologique est en cela utilisé avec force. En effet, comment ne pas vouloir habiter « une maison où l'air est sain » (publicité Leroy Merlin)?

\title{
Conclusion : l'être-au-monde face à ses choix... contraints
}

Puisque c'est l'homme qui constitue son monde, c'est aussi lui qui fait les choix du Monde. Le déterminisme se trouve ici finalement inversé par rapport aux représentations du 19e siècle. Ce n'est plus le contexte physique, naturel, social, économique qui détermine les façons d'être, de faire et de penser des êtres humains, mais ce sont les choix individuels qui co-déterminent les contextes dans lesquels nous nous trouvons tous. Il faut donc valoriser cette autonomisation prétendue de la 
décision. Et en matière de consommation, ce choix éthique, durable, responsable, authentique n'en est que plus valorisé.

Banquier d'une planète vivable. Au service de l'environnement, de l'éco-habitat, des énergies renouvelables, alors que la planète se réchauffe, que le pétrole flambe, justement, Faut le faire. Crédit coopératif, C'est un choix. (Groupe Banque Populaire)

L'autonomie de la décision est présentée comme une règle. Le site de France Nature Environnement propose une rubrique intitulée « j'agis ». En matière de développement durable, c'est par la somme des initiatives individuelles que l'on viendra à bout des problèmes d'environnement. C'est sur ce fondement, érigé en dogme par la publicité, que se construit l'éducation à l'environnement pour un développement durable. Dans cet objectif de conscientisation et de sensibilisation individuelle aux pratiques vertueuses, l'individu est la cible marketing. "C'est la somme des petits gestes pour l'environnement qui peut venir à bout des problèmes ».

C'est l'un des objectifs affichés par le logo « La biodiversité, c'est ma nature » (figure 1) proposé à l'occasion de l'année internationale de la biodiversité par un collectif d'ONG (WWF, LPO, UICN, Nicolas Hulot, FNE, Ligue Roc) censé défendre l'environnement. Le jeu rhétorique est ici très clair : une passerelle s'effectue entre ma nature au sens d'une nature naturelle et ma nature au sens de ce que je suis fondamentalement à l'intérieur de mon Être, donc entre l'environnement qui m'entoure (mis en lumière ici par l'ensemble des éléments symboliques censés évoquer cet environnement "biodivers ", végétaux et animaux divers) et ce qui initie ce que je suis, ce que je fais, ce que je pense dans celui-ci. Cela évoque tout à la fois le choix inhérent de l'habitant qui est au centre de cette nature, seul au monde, tel Adam au jardin d'Eden, mais aussi l'idée d'une détermination de cet habitant par ce qui l'entoure, comme s'il allait déteindre, reprendre des couleurs à l'aune de l'aspect multicolore de l'environnement. La cible, c'est l'individu habitant du monde. Le message éducatif a changé d'origine.

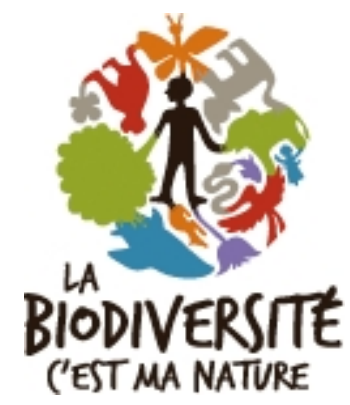

Figure 1 : Logo « La biodiversité, c'est ma nature »

Il est en effet assez remarquable de constater la moindre place prise par l'éducation nationale qui, elle, incarne l'instruction de masse, le projet collectif de société. Il ne s'agit pas d'incorporer dans les objectifs de l'école des programmes d'éducation à l'environnement, le développement durable est bien entré dans les programmes de SES (Sciences Économiques et Sociales), mais à la marge. Cette incapacité à s'adapter ou cette défaillance est compensée par la Fondation Nicolas Hulot, par exemple, qui propose " une autre école " ${ }^{2}$, dont "la mission est dédiée à la protection du vivant ", comme si la mission de l'école pour tous n'était pas la protection du vivant. La mission 
éducative cède la place aux "prestations offertes" par la fondation qui propose un partenariat original « privé public ».

Le relais des pouvoirs publics est flagrant à travers des campagnes de sensibilisation qui s'adressent désormais aux individus et non plus au collectif faisant société. L'État, comme les entreprises et même le monde associatif (ex.: France Nature Environnement), interpelle l'homme à l'occasion des plages de publicité radiophoniques en lui racontant une histoire: celle de ce vieil aspirateur qui a beaucoup servi et ne souhaite pas être abandonné dans une décharge, mais bien remis au magasin fournisseur du nouvel aspirateur qui se chargera de le recycler, le développement durable ne devant pas être un message de décroissance. Et le spot se termine inlassablement par la formule suivante : «Ceci est un message du ministère de l'Écologie, du Développement durable, des Transports et du Logement ». Ce n'est pas par l'action et la mobilisation du collectif (à travers l'impôt l'aménagement ou la règle) que l'État s'empare du problème des déchets, mais par la communication.

Il y a donc une convergence assez forte sur la méthode entre les pouvoirs publics, le monde associatif et l'entreprise. Il s'agit d'exalter l'individu dans une conception néolibérale de la société qui valorise l'initiative habitante, l'entreprise individuelle, la réussite personnelle. La phénoménologie, par la compréhension simpliste et caricaturale qui en est faite, permet alors de donner des bases philosophiques à cet individualisme. Celui d'un habitant qui vit de projets, qui pense construire ses choix, ses initiatives, ses actions. Mais au-delà de ces projets, n'y a-t-il pas avant tout des marchandises à vendre à cet être éco-responsable, marchandises noyées dans la rhétorique techniciste de l'innovation éco-environnementale utilisant les éléments de la nature? Rhétorique dont les tenants et aboutissants économiques étouffent le questionnement même sur la technique alors que ce questionnement est la base du dévoilement de l'habitation et donc de la compréhension de l'être humain contemporain (Heidegger, [1954] 1958).

\section{BIBLIOGRAPHIE}

Bernays, E. ([1928] 2007). Propaganda. Comment manipuler l'opinion en démocratie. Paris : La

Découverte (Zones).

Bofill, R. (1989). Espaces d'une vie. Paris : Éditions Odile Jacob.

Bourdieu, P. (1994). Raisons pratiques. Sur la théorie de l'action. Paris : Seuil.

Buttimer, A. (1980). Home, Reach, and the Sense of Place. In Buttimer, A. and Seamon, D. (Eds.).

The Human Experience of Space an Place (p. 166-187). London : Croom Helm.

Caron, J. (2010). Quartiers brisés, habitants spoliés. Paris : Non Lieu.

Chrétien, J.-L. (1983). De l'espace au lieu. In Damian, H. et Raynaud, J.-P. (dir.). Les symboles du lieu : l'habitation de l'homme (p. 117-138). Paris : Les Éditions de l'Herne. 
Degoutin, S. (2006). Prisonniers volontaires du rêve américain. Paris : Éditions de la Villette. Di Méo, G. (1998). Géographie sociale et territoires. Paris : Nathan.

Ferrié, C. (1999). Heidegger et le problème de l'interprétation. Paris : Éditions Kimé. Franck, D. (1986). Heidegger et le problème de l'espace. Paris : Les Éditions de Minuit. Giddens, A. (1987). La constitution de la société. Paris : PUF.

Heidegger, M. ([1927] 1964). L'Être et le Temps. Paris : Gallimard.

Heidegger, M. ([1946] 1953-1983). Lettre sur l'humanisme. Paris : Aubier-Montaigne. Heidegger, M. (1958). Essais et Conférences. Paris : Gallimard.

Hoyaux, A.-F. (2002). Entre construction territoriale et constitution ontologique de l'habitant : Introduction épistémologique aux apports de la phénoménologie au concept d'habiter. Cybergeo : European Journal of Geography, 216, http://cybergeo.revues.org/1824

Hoyaux, A.-F. (2005). L'ubiquité publicitaire. In Volvey, A. (dir.). Échelles et temporalités (p. 160-164). Paris : Atlande.

Le Corbusier (1957). La Charte d'Athènes. Paris : Les Éditions de Minuit.

Lévy, J. et Lussault, M. (2003). Dictionnaire de géographie et de l'espace des sociétés. Paris : Belin. Lussault, M. (2003). Acteur. In Lévy, J. et Lussault, M. Dictionnaire de géographie et de l'espace des sociétés (p. 39-42). Paris : Belin.

Paquot, T., Lussault, M. et Younès, C. (dir.) (2007). Habiter, le propre de l'humain. Villes, territoires et philosophie. Paris : La Découverte.

Patocka, J., ([1960-1976] 1988). Qu'est-ce que la phénoménologie ? Grenoble : Éditions Jérôme Millon. Ricœur, P. (1985). Temps et récit. Tome 3 : Le temps raconté. Paris : Éditions du Seuil. Schütz, A. ([1942-1966] 2007). Essais sur le monde ordinaire. Paris : Le Félin Poche. Serres, M. (1999). Le contrat naturel. Paris : Flammarion.

Staulber, J. et Rampton, S. (2004). L'industrie du mensonge. Lobbying, communication, publicité \& médias. Marseille : Agone.

\section{NOTES}

1. L'ensemble du corpus documentaire est consultable à l'adresse suivante: http:// critiquesdepub.blogspot.com/

2. Pour plus de détails, consulter le site http://www.ecole-nicolas-hulot.org/ecole/projet/ projet.php

\section{RÉSUMÉS}

Ce texte éclaire la récupération médiatique effectuée par la publicité autour du concept d'habiter abordé par la phénoménologie. En effet, la communication publicitaire semble jouer et se jouer des approches présentiste (tout relève du ici et maintenant) et constitutiviste (tout est construit 
en acte par l'habitant hors d'une quelconque objectivation rationnelle de la réalité) développées par ce courant philosophique pour en détourner les valeurs positives au regard des habitants de nos sociétés contemporaines. Sous les vocables projet, intentionnalité, authenticité, habiter, la publicité met en scène et en récit l'individu éco-responsable de la nature et du monde qu'il construit, qu'il invente ici et maintenant en fictions pour les générations futures! Elle valorise alors subtilement les choix «d'acteurs » en rendant prétendument à l'habitant sa puissance réflexive de décision, de démocratie, alors qu'à travers son message même, elle le dépossède déjà de son esprit critique pour le conformer à un contexte ambiant techniciste plus préoccupé de progrès que de réflexivité.

This article discusses the mediatic utilization of the phenomenological concept of marketing surrounding the concept of inhabiting. In effect, it appears that marketing communication is playing with and around two approaches related to that philosophical trend: the presentist (everything is about being here and now) and the constitutivist (everything is about the inhabitant's construction of reality, no rational objectivation is considered). In doing so, marketing comunication is taking away the positive values associated with the concept of inhabiting in its genuine, philosophical sense. Using words such as " project ", "intentionality ", " authenticity" and " inhabit", marketing invents individuals who are eco-responsible towards nature and towards the world they are building in fiction... for future generations! Marketing subtely places importance on certain choices by actors, those taking advantage of their reflexive and democratic attributes, while it is in fact taking away the critical spirit of the viewers; it wants them to conform to a technicist ambiance where the concern is about progress much more than about reflexivity.

\section{AUTEURS}

\section{VÉRONIQUE ANDRÉ}

Agrégée de géographie, Véronique André est enseignante chercheure à l'UMR ADES CNRS/ Université de Bordeaux. Ses travaux de recherches portent sur la construction des représentations de la nature et l'analyse de modes de gestion de l'environnement, et notamment sur les modalités d'élaboration de l'action publique environnementale.

\section{LAURENT COUDERCHET}

Enseignant chercheur en géographie à l'UMR ADES CNRS/Université de Bordeaux, ses travaux portent sur les paysages et la reconnaissance géomatique des états de la nature : diagnostics experts, diagnostics profanes partagés. Discours et politiques publiques de préservation de la biodiversité participent de ses centres d'intérêt.

\section{ANDRÉ-FRÉDÉRIC HOYAUX}

Enseignant-chercheur à l'UMR ADES CNRS/Université de Bordeaux, à l'aide d'analyses qualitatives effectuées sur divers types de discours (verbaux, iconiques, filmiques), AndréFrédéric Hoyaux travaille sur les constructions idéologiques, identitaires, territoriales qu'opèrent les acteurs à partir et à travers différentes figures et catégories spatiales. 